\title{
Burst-by-burst Adaptive Joint-Detection CDMA/H.26L Based Wireless Video Telephony using TTCM and LDPC Codes
}

\author{
J. Y. Chung, F. Guo, S. X. Ng and ${ }^{1}$ L. Hanzo \\ Dept. of ECS, University of Southampton, SO17 1BJ, UK. \\ Tel: +44-23-8059 3125, Fax: +44-23-8059 4508 \\ Email: ${ }^{1}$ lh@ecs.soton.ac.uk, http://www-mobile.ecs.soton.ac.uk
}

\begin{abstract}
A low bit-rate video coding techniques using the H.26L standard codec for robust transmission in mobile multimedia environments are presented. For the sake of achieving error resilience, the source codec has to make provisions for error detection, resynchronization and error concealment. Thus a packetization technique invoking adaptive bit-rate control was used in conjuction with the various modulation scheme employed. In this contribution, we propose a Burst-by-Burst Adaptive Coded Modulation-Aided Joint Detection-Based CDMA (ACM-JD-CDMA) scheme for wireless video telephony and characterise its performance when communicating over the UTRA wideband vehicular fading channels. The coded modulation schemes invoked in our fixed modulation mode based systems are Low Density Parity Check code based Block Coded Modulation (LDPC-BCM) and Turbo Trellis Coded Modulation (TTCM). The performance of LDPC-BCM was evaluated and compared to that of TTCM in the context of the ACM-JD-CDMA system using a practical modem mode switching regime. Both schemes exhibited a similar transmission integrity, although the LDPC arrangement is capable of achieving this at a lower complexity.
\end{abstract}

\section{INTRODUCTION}

In classic digital communication systems, modulation and errorcorrection coding are typically considered as two different entities. However, the combination of error-correction coding with modulation leads to the attractive concept of Trellis Coded Modulation (TCM) [1, 2]. TCM is a bandwidth efficient scheme, where the redundancy introduced by channel coding does not expand the required bandwidth, since the parity bits are absorbed by the extended signal constellation. Turbo TCM (TTCM) is another bandwidth efficient scheme that has a structure similar to that of the family of power-efficient binary turbo codes [3], but employs TCM schemes as component codes [2,4]. It has been shown in [5] that TTCM is capable of outperforming other coded modulation schemes in the context of H.263 based video telephony [6], when communicating over the UMTS Terrestrial Radio Access (UTRA) wideband vehicular fading channel [7,8], while maintaining a comparable decoding complexity.

Another powerful channel coding scheme involved in our study is the family of Low Density Parity Check (LDPC) codes, which were devised by Gallager [9] in 1962. LDPC codes belong to the class of linear block codes, which are defined by a parity check matrix having $M$ rows and $N$ columns. The column weight and row weight is low compared to the dimension $M$ and $N$ of the parity check matrix. During the early evolutionary phase of channel coding, LDPC schemes made limited impact on the research of the channel coding community, despite their impressive performance, which was unprecedented prior to the turbo coding era. This lack of popularity was a consequence of its relatively high storage requirement and complexity. However, in recent years research interests in LDPC codes have been rekindled owing to their capability of approaching Shannon's predicted performance limits. In order to lend the LDPC codes a high spectral efficiency, a LDPC-based Block Coded Modulation (LDPC-BCM) scheme was proposed in [10]. When employing a long codeword length of 3000 modulated symbols, regular LDPC-BCM was found to slightly outperform TTCM in the context of non-dispersive uncorrelated Rayleigh fading channels at a similar coding rate and a comparable decoding complexity [11].

In order to counteract the time varying nature of mobile radio channels, in this study Adaptive Coded Modulation (ACM) was employed[12]. Explicitly, a higher order modulation mode is employed, when the instantaneous estimated channel quality is high, in order to increase the number of Bits Per Symbol (BPS) transmitted. Conversely, a more robust lower order modulation mode is employed, when the instantaneous channel quality is low, in order to improve the mean Packet Loss Ratio (PLR) performance. The Minimum Mean Square Error Decision Feedback Equalizer (MMSE-DFE) based Joint Detector (JD-MMSE-DFE) is invoked in the proposed Code Division Multiple Access (CDMA) [13] ACM scheme. Specifically, joint detection $[13,14]$ receivers are derivatives of the well-known single-user equalizers, which were originally designed for equalizing signals that have been corrupted by Inter-Symbol Interference (ISI) due to the multipath effect of wireless channels. The JD-MMSE-DFE scheme constitutes a powerful approach to mitigating the effects of both Multi-User Interference (MUI) and ISI [13], while at the same time improving the system's performance by benefiting from the multipath diversity gain provided by the dispersive channel.

In this contribution, a Burst-by-Burst ACM-aided Joint DetectionBased CDMA (ACM-JD-CDMA) scheme is proposed for H.26L/H.263 based wireless video telephony [15]. The system is characterised in performance terms when communicating over the uplink (mobile stationto-base station) UTRA [13] wideband vehicular fading channel. Specifically, regular LDPC-BCM is studied and compared to TTCM in the context of the proposed ACM-JD-CDMA system.

\section{SYSTEM OVERVIEW}

We invoke four different-rate channel encoders in the context of the TTCM/LDPC-BCM scheme, each adding one parity bit to each information symbol, yielding a coding rate of $1 / 2$ in conjunction with the modulation mode of 4QAM, $2 / 3$ for 8PSK, $3 / 4$ for 16QAM and $5 / 6$ for 64QAM. The TTCM scheme invoked Robertson's codes [4] and the Log-Maximum A Posteriori (Log-MAP) decoding algorithm [16] was utilised. The regular LDPC-BCM scheme was designed by adjusting the row weight of its generator matrix, in order to arrive at the desired coding rate, while stipulating a fixed column weight of three [10]. The decoding algorithm employed was the sum-product algorithm [17]. For the sake of a fair comparison, the parameters of TTCM and LDPC-BCM were adjusted such that their decoding complexity became similar. Explicitly, TTCM schemes employing TCM 
codes of memory three were invoked for the various modulation modes in conjunction with a fixed number of decoding iterations, namely four. For the sake of maintaining a similar complexity, the column weight of LDPC-BCM was fixed to three and the maximum number of decoding iterations of the LDPC-BCM scheme was set to 15, 10, 10 and 17 in conjunction with 4QAM, 8PSK, 16QAM and 64QAM, respectively [10].

At each mobile station, the information bits generated by the user's video encoder are first channel encoded in order to generate non-binary symbols according to the specific Coded Modulation (CM) mode chosen by the base-station on the basis of the instantaneous channel quality encountered, as suggested in [13]. The CM-encoded symbols are then direct-sequence spread with the aid of the CDMA multipath spreading code assigned to the user, modulated on to the carrier and transmitted over the dispersive UTRA channels. At the receiver of the basestation, the data symbols of all users are jointly detected by the JDMMSE-DFE and the detected symbols are channel decoded by the corresponding $\mathrm{CM}$ decoder, before they are processed by the video decoder.

In joint detection systems the SINR of each user recorded at the output of the MMSE-DFE can be calculated by using the channel estimates and the spreading sequences of all the users. By assuming that the transmitted data symbols and the noise samples are uncorrelated, the analytical expression derived for calculating the SINR, $\gamma_{o}$, of the $n$-th symbol transmitted by the $k$-th user was given by Klein $e t$ al $[13,18]$ as:

$$
\begin{aligned}
\gamma_{o}(j) & =\frac{\text { Wanted Signal Power }}{\text { Res. MAI and ISI Power + Eff. Noise Power }} \\
& =g_{j}^{2}[\mathbf{D}]_{j, j}^{2}-1, \quad \text { for } j=n+N(k-1),
\end{aligned}
$$

where SINR is the ratio of the wanted signal power to the residual MAI and ISI power plus the effective noise power. The number of users in the system is $K$ and each user transmits $N$ symbols per transmission burst. The matrix $\mathbf{D}$ in Eq (1) is a diagonal matrix that is obtained with the aid of the Cholesky decomposition [19] of the matrix used for linear MMSE equalization of the CDMA system [13,18]. Furthermore, the notation $[\mathbf{D}]_{j, j}$ in Eq (1) represents the element in the $j$-th row and $j$-th column of the matrix $\mathbf{D}$ and the value $g_{j}$ is the amplitude of the $j$-th symbol.

After the output SINR value of each users is estimated at the base station, the best-matching modulation mode capable of maintaining the required target integrity, while achieving the highest possible effective video throughput is chosen accordingly and communicated to the corresponding mobile stations by superimposing this ACM-mode side information on the downlink (base station-to-mobile station) transmission burst. In our practical approach, imperfect rather than perfect channel estimation was employed in the adaptive signalling mechanism. More explicitly, the channel quality estimate obtained by the mobile station from the base station is delayed by $4.615 \mathrm{~ms}$, i.e. by one UTRA frame duration owing to the associated signalling delay.

Let us denote the choice of modulation mode by $V_{m}$, where the total number of modulation modes is $M=4$ and $m=1,2, \ldots, M$. The ACM/CDMA modulation mode having the lowest number of constellation points is $V_{1}$ and the one associated with the highest throughput is $V_{M}$. The rules used for switching the modulation modes are as follows:

$$
\begin{array}{ccc}
\Gamma_{o}(k)<t_{1} & \Longrightarrow & V_{1}=4 Q A M \\
t_{1} \leq \Gamma_{o}(k)<t_{2} & \Longrightarrow & V_{2}=8 P S K \\
t_{2} \leq \Gamma_{o}(k)<t_{3} & \Longrightarrow & V_{3}=16 Q A M \\
t_{3} \leq \Gamma_{o}(k) & \Longrightarrow & V_{4}=64 Q A M,
\end{array}
$$

where $\Gamma_{o}(k)$ is the SINR of the $k$-th user at the output of the MMSE-
DFE, which was calculated by using Equation 1 and

$$
\Gamma_{o}(k)=\frac{1}{N} \sum_{n=1}^{N} \gamma_{o}(j), \quad j=n+N(k-1) .
$$

The values $\left(t_{1}, \cdots, t_{M-1}\right)$ represent the switching thresholds used for activating the ACM/CDMA modulation modes, where we have $t_{1}<t_{2}<\cdots<t_{M-1}$. In our proposed system, the ACM/CDMA switching thresholds were chosen such that the resultant PLR became lower than $5 \%$ and the system's video throughput was maximised.

\section{VIDEO OVERVIEW}

\begin{tabular}{|l||c|c|c|c|}
\hline \multicolumn{1}{|l||}{ Features } & \multicolumn{4}{c|}{ Multi-rate System } \\
\hline \hline \multicolumn{1}{|l||}{ Mode } & 4QAM & 8PSK & 16QAM & 64QAM \\
\hline Transmission Symbols & \multicolumn{5}{c|}{240} \\
\hline Bits/Symbol & 2 & 3 & 4 & 6 \\
\hline Transmission bits & 480 & 720 & 960 & 1440 \\
\hline Packet Rate & \multicolumn{5}{|c|}{$100 / \mathrm{s}$} \\
\hline Transmission bitrate (kbit/s) & 48 & 72 & 96 & 144 \\
\hline Data Symbols & \multicolumn{5}{|c|}{234} \\
\hline Coding Rate & $1 / 2$ & $2 / 3$ & $3 / 4$ & $5 / 6$ \\
\hline Information Bits/Symbol & 1 & 2 & 3 & 5 \\
\hline Unprotected bits & 240 & 480 & 720 & 1200 \\
\hline Unprotected bitrate (kbit/s) & 24.0 & 48.0 & 72.0 & 120.0 \\
\hline Video packet CRC (bits) & \multicolumn{4}{|c|}{16} \\
\hline Feedback protection (bits) & \multicolumn{5}{|c|}{9} \\
\hline Video packet header (bits) & 11 & 12 & 12 & 13 \\
\hline Video bits/packet & 204 & 443 & 683 & 1162 \\
\hline Effective Video-rate (kbit/s) & 20.4 & 44.3 & 68.3 & 116.2 \\
\hline Video framerate (Hz) & & \multicolumn{4}{|c}{30} \\
\hline
\end{tabular}

TABLE I

OPERATIONAL-MODE SPECIFIC TRANSCEIVER PARAMETERS FOR LDPC.

\section{A. ITU-T VCEG project H. $26 L$}

In this study, we transmitted 176x144-pixel Quarter Common Intermediate Forma $\mathrm{t}$ (QCIF) resolution video sequences at 30 frames/s using a reconfigurable TDD/CDMA transceiver, which can be configured as a 2, 3, 4 or 6 bit/symbol scheme. The proposed video transceiver is based on the H.26L video codec [15].

The H.26L codec [15] was devised by the ITU-T Video Coding Expert Group (VCEG). The video codec is still under development. This paper refers to the version described in the Test Model Long Term Number 8 (TML-8) [20]. The H.26L video coding layer's (VCL) algorithm has a design similar to that of the ratified video coding standards, such as those specified by the ISO MPEG visual and the ITU-T standards. In addition, it contains new features that enable it to achieve a significant improvement in compression efficiency in relation to the previously ratified coding standards.

Every input Macroblock (MB) has to be predicted in the H.26L scheme before the transform coding process. Sub-blocks of $4 \times 4$ samples are used for transform coding. The conventional picture types known as Intra-frame coded (I), Inter-frame coded or Prediction (P) based and Bidirectional (B) prediction aided coding modes are still supported. There are two classes of Intra-frame coding modes, which are referred to as INTRA $16 \times 16$ and INTRA $4 \times 4$ modes. Moreover, in the H.26L coding scheme prediction is always utilized in the spatial domain by referring to the neighbouring video pixels of already coded blocks. When the INTRA $4 \times 4$ mode is used, each $4 \times 4$-pixel block of luminance samples utilizes one of the six proposed prediction modes [15]. The identifiers of the prediction modes chosen are then transmitted to the decoder as side-information. For picture regions having less spatial detail, the Intra $16 \times 16$ prediction mode is 
employed, in which one of four prediction modes is chosen for the prediction of the entire MB [15].

As for the Inter-frame coded prediction modes, H.26L provides seven motion-compensated coding modes for the MBs of Inter-frame coded (P) pictures. Each motion compensated mode corresponds to a specific partitioning of the MB into fixed-size blocks used for enhancing the achievable quality of motion description. Currently, block sizes of $16 \times 16,16 \times 8,8 \times 16,8 \times 4,4 \times 8$ and $4 \times 4$ pixels are supported by the H.26L syntax [15], and thus up to 16 motion vectors can be transmitted for a MB. The so-called spiral search aided motion compensation procedure [15] finds the 'minimum cost' coding solution for each block size within a specified search window. The most popular block-matching measure in this context is the Sum of Absolute Difference (SAD) [15]. The 'cost' includes the SAD-based coding distortion and the corresponding overhead bits required for encoding the block size information and the motion vectors. The optional block size is decided based on finding the minimum cost solutions. If a $4 \times 4$ block size is the 'winner', there are 16 motion vectors for the entire MB. The resolution of the motion vectors is at least a quarter of a pixel. When requiring a higher coding efficiency, the H.26L codec allows 1/8-pixel accuracy motion prediction, although this imposes a high encoding complexity.

The motion prediction residual is transformed to the spatial frequency domain using $4 \times 4$ pixel integer-valued DCT. Instead of the conventional two-dimenssional (2D) DCT, H.26L uses a separable vertical and horizontal DCT, processing integer values while maintaining a similar performance to the conventional 2D 4x4 DCT. More explicitly, both the DCT and the inverse transform use exact integer operations, and hence the detrimental effects of quantization errors are avoided [15]. The scanning order of the DCT coefficients is still based on zig-zag scanning, similarly to that used for example in the H.263 standard [8]. There are two different entropy coding techniques that are used in the H.26L codec for compressing the quantized DCT coefficients, namely the Universal Variable Length Codes (UVLC) [15] and Context-Based Adaptive Binary Arithmetic Coding (CABAC) modes [15]. The UVLC scheme provides a simple and robust method for encoding all side-information and the DCT coefficients. However, its achievable performance is modest at moderate and high bit rates, where the CABAC mode performs better [15].

\section{B. Packetization in Mobile Environment}

Let us now consider the video packetisation algorithm. The video packet size varies in response to the current operating mode of the multi-mode modem. The proposed multi-mode system can be configured to switch amongst the 2, 3, 4 and $6 \mathrm{bit} / \mathrm{symbol}$ modulation schemes based upon the near-instantaneous channel conditions. Again, as shown in Table I, when the channel is benign, the video bitrate is approximately $116 \mathrm{Kbps}$. However, as the channel quality degrades, the modem will switch to the 4QAM mode of operation, where the video bitrate drops to $20 \mathrm{Kbps}$.

The video transmitter is informed of the packet's transmission success or failure with the aid of a highly protected feedback channel, which is integrated into the strongly-protected reverse link. More explicitly, the associated feedback flag is protected with the aid of a repetition code and concatenated to the reverse-direction information packets. The use of packet acknowledgements allows the video encoder and the remote decoder to keep 'synchronized', i.e. to operate on the basis of identical reconstruction frame buffer contents without the need of video packet retransmissions, which are wasteful in terms of bandwidth efficiency and transmission delay.

\begin{tabular}{|l|r|}
\hline Parameter & Value \\
\hline \hline Carrier Frequency & $1.9 \mathrm{GHz}$ \\
\hline Vehicular Speed & $30 \mathrm{mph}$ \\
\hline Doppler frequency & $85 \mathrm{~Hz}$ \\
\hline System Baud rate & $3.84 \mathrm{MBd}$ \\
\hline Normalised Doppler frequency & 85Hz/3.84MBd=2.21 $\times 10^{-5} \mathrm{~Hz}$ \\
\hline Channel type & UMTS Vehicular Channel A \\
\hline Number of paths in channel & Adaptive Coded Modulation \\
\hline Data modulation & JQAM, 8PSK, 16QAM, 64QAM) \\
\hline Receiver type & JD-MS-DFE \\
\hline No. of symbols per JD block & 20 \\
\hline
\end{tabular}

TABLE II

MODULATION AND CHANNEL PARAMETERS

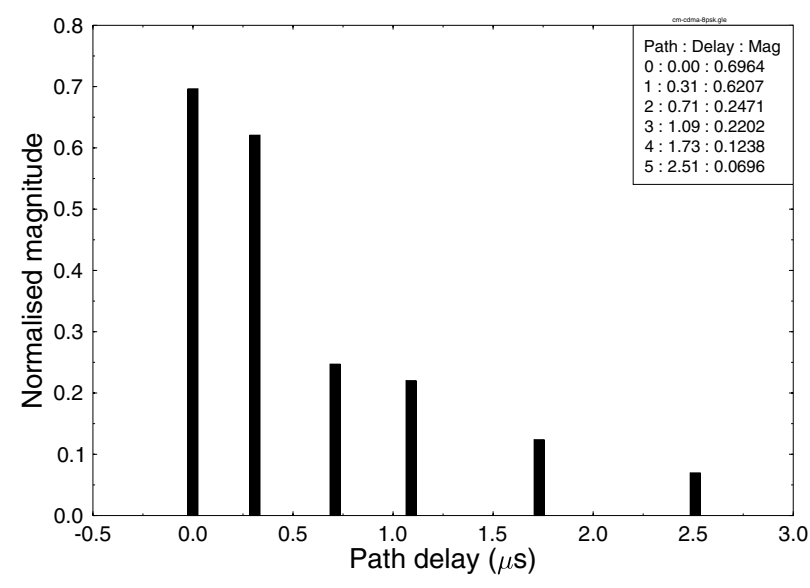

Fig. 1. UTRA vehicular channel A [7].

\section{Channel Model and System Parameters}

The associated video system parameters are summarized in Table II. The multi-path channel model is characterized by its discretised chipspaced UTRA vehicular channel A [7]. The corresponding channel impulse response is shown in Figure 1, where each path is faded independently according to the Rayleigh distribution. The transmission burst structure of the modified UTRA Burst 1 [8] using a CDMA spreading factor of eight is shown in Figure 2. For the sake of maintaining a low MUD complexity, the number of data symbols per JD block was set to 20 , hence the original UTRA Burst 1 was slightly modified to host a burst of 240 data symbols, which is a multiple of 20. Furthermore, the number of slots per frame is 15 , and the frame duration is $10 \mathrm{~ms}$. For the sake of benchmarking, the remaining system parameters were the same as in [5].

\section{EXPERIMENTAL RESUlts AND DiscusSiONS}

Simulations were performed using the QCIF-format head-and-shoulder "Miss America" videophone sequence. The various modulation modes of Table I were used. Figure 3 shows the Packet Loss Ratio (PLR) versus channel SNR performance for each mode of the system. Our video scheme discards all video packets, which are not error-free,

2/3 ms, 2560 chips, 240 data symbols

\begin{tabular}{|c|c|c|c|}
\hline \multicolumn{3}{|c|}{} \\
\hline 120 data symbols & Midamble & 120 data symbols & $\begin{array}{c}\text { Guard } \\
960 \text { chips }\end{array}$ \\
544 chips & 960 chips & 96 chips \\
\hline
\end{tabular}

Fig. 2. A modified UTRA Burst 1 [8] with a spreading factor of 8 . The original UTRA burst has 244 data symbols. 
hence the PLR is a more accurate measure of the expected video performance than the BER, which is zero. Needless to say, our goal is to maintain as low a PLR as possible. Again, it should be noted that for channel SNRs in excess of $25 \mathrm{~dB}$ the 64QAM mode offered an acceptable packet loss ratio of less than $5 \%$, providing a video rate of approximately $113 \mathrm{Kbps}$. However, as the channel quality degrades, the multi-mode system is switched to the lower rate modes of 16QAM, then to 8PSK and eventually to 4QAM.

Having shown the effects of the channel's SNR on the PLR, let us now demonstrate these effects on the decoded video quality measured in terms of the Peak-Signal-to-Noise Ratio (PSNR). The available video quality of our video system is directly related to the PLR performance. Figure 4 shows the associated average PSNR versus channel SNR performance, demonstrating that at high channel SNRs the fixed modulation modes of the adaptive signalling regime exhibit a higher PSNR in conjuction with the higher-throughput modulation modes, than in the lower-throughput modulation mode. For example, at high SNRs 64QAM yields a PSNR of 43.60dB, whereas the 4AQM mode achieves a PSNR of 37.10dB.

The video quality of each of the fixed modes degrades dramatically, as the PLR is increased, which results in parts of the picture being 'frozen' for one or possibly several consecutive video frames. This phenomena is illustrated in Figure 5.

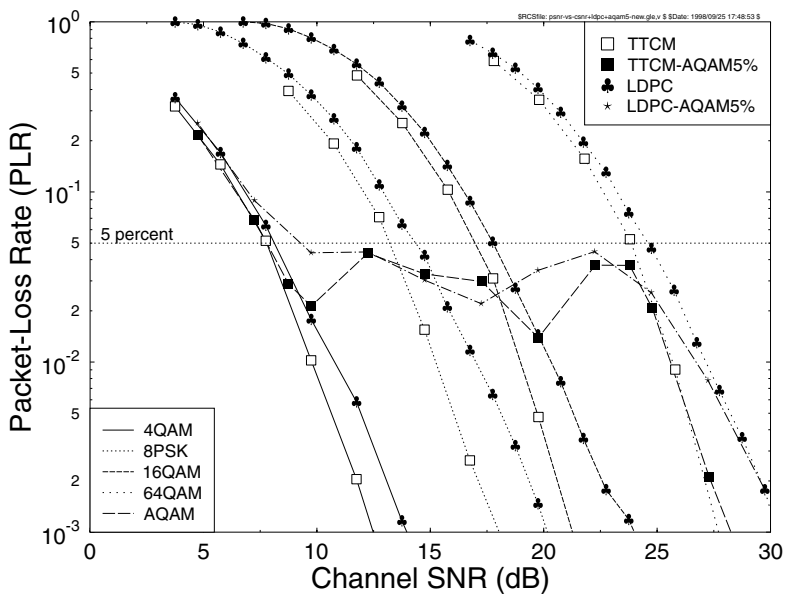

Fig. 3. Packet loss ratio versus channel SNR for the four fixed modem modes, using the TTCM and LDPC joint coding/modulation schemes considered, when communicating over the UTRA channel of Figure I.

As for the adaptive system, it can be seen from Figure 4 that the video quality degrades gracefully, when the system gradually switches from the high-throughput modes to the lower-throughput modes. The LDPC-based AQAM curve of Figure 4 has been repeated in Figure 6 for comparison. Here, we demonstrate the additional performance gains that are achievable, when TTCM coding is used in preference to the LDPC coding. Observe in Figure 6 that the overall performance of the TTCM assisted H.26L video codec is slightly higher than that of the LDPC aided H.26L video codec. These results were recorded at a similar decoding complexity, when a short codeword length of 240 modulated symbols was employed by both schemes. Note however that LDPC coding has been shown in [11] to slightly outperform TTCM at a given decoding complexity, when a long codeword length is invoked, while communicating over a fast-fading/uncorrelated channel.

For the sake of further benchmarking, our results recorded for a similar TTCM ACM/CDMA system using the H.263 codec [5] are also depicted in Figure 6. These results show that under the same channel conditions and using the same system parameters, the H.26L codec

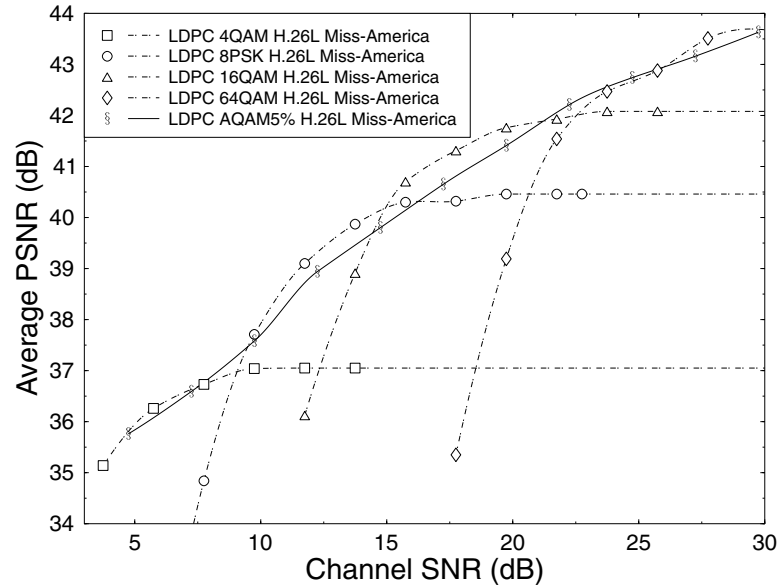

Fig. 4. Average PSNR versus channel SNR for the four fixed modes and for the LDPC-based ACM/CDMA scheme using the QCIF Miss America video sequence at $30 \mathrm{frame} / \mathrm{s}$.

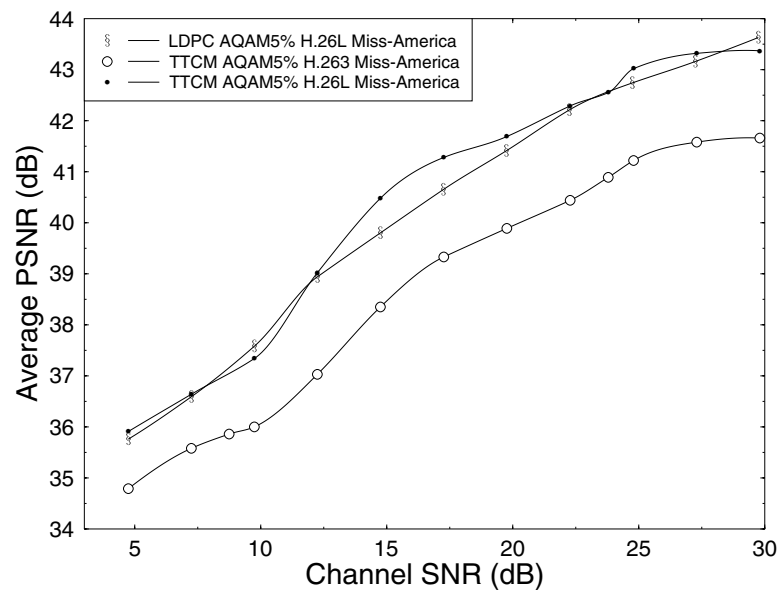

Fig. 6. Average PSNR versus channel SNR for the LDPC and TTCM CDMA AQAM schemes at PLR $=5 \%$.

based system outperformed the H.263 coding aided arrangement.

The video quality versus PLR performance can be more explicitly observed in Figure 7. The figure shows how the video quality degrades, as the PLR increases. Observe that in order to ensure a seamless degradation of the video quality as the channel SNR reduced, it was beneficial to switch to a more robust modulation mode, when the PLR exceeded 5\%. Although this inherently reduced the effective video bitrate and the associated video PSNR, the video degradation was less objectionable in subjective video quality terms, than inflicting a PLR in excess of $5 \%$ would have been.

\section{CONCLUSIONS}

As a conclusion, various burst-by-burst adaptive coded modulation aided joint detection based CDMA video transceivers have been studied, when communicating over the UTRA wideband vehicular fading channel.

A burst-by-burst adaptive ACM/CDMA modem maximizes the system's throughput by using the most appropriate modulation mode in response to the instantaneous channel conditions. Furthermore, we have quantified the achievable video performance gains as a benefit of employing the proposed burst-by-burst adaptive CDMA modem.

The burst-by-burst adaptive modem guaranteed the same video performance, as the lowest- and highest-order fixed-mode modulation sch- 


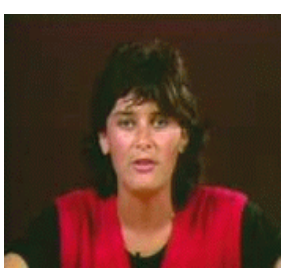

Frame \#60

Error Free Frame

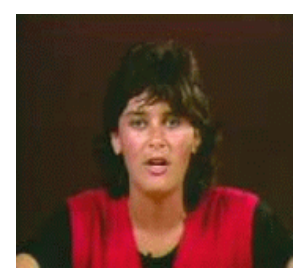

Frame \#61

Error Free Frame

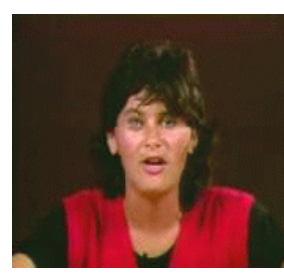

Frame \#62

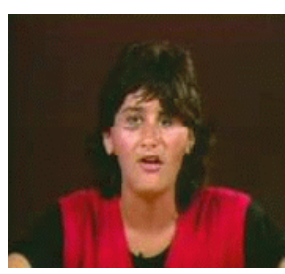

Frame \#63

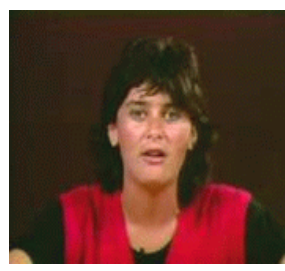

Frame \#64

Recovered Video Frame

TIME

Fig. 5. The channel has corrupted some of the transmitted packets resulting in part of the video image become frozen as shown in frames62 and 63 . However the system soon recovers and replenishes the affected areas as in frame 64 .

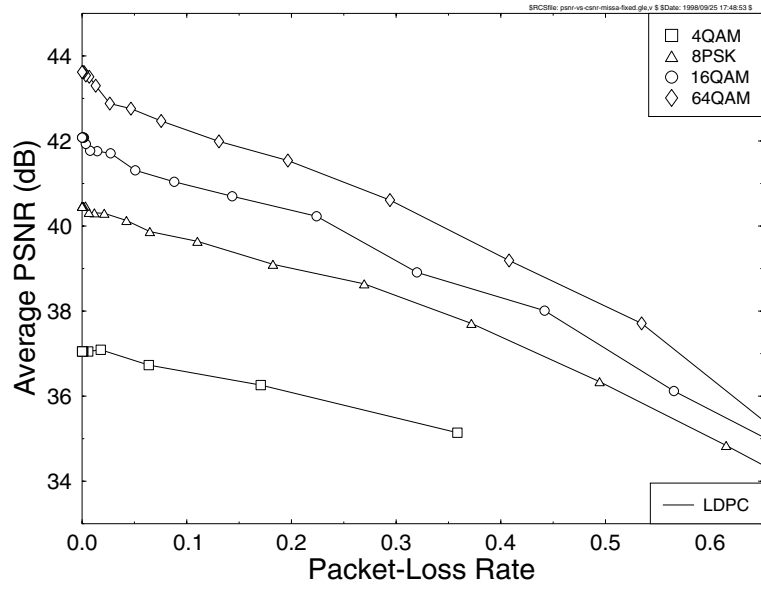

Fig. 7. Decoded video quality (PSNR) versus video packet loss ratio for the four modulation modes.

emes at a range of low and high channel SNRs. However, between these extreme SNRs the effective video bitrate gracefully increased, as the channel SNR increased, whilst maintaining a near-constant PLR. By controlling the AQAM switching thresholds a near-constant PLR can be maintained. Finally, as shown in Figure 6, the H.26L video codec outperformed the H.263 codec in video PSNR terms.

\section{ACKNOWLEDGEMENTS}

The financial support of the European Union and that of the EPSRC, Swindon UK is gratefully acknowledged.

\section{REFERENCES}

[1] G. Ungerböck, "Channel coding with multilevel/phase signals," IEEE Transactions on Information Theory, vol. 28, pp. 55-67, January 1982.

[2] L. Hanzo, T.H. Liew and B.L. Yeap, Turbo Coding, Turbo Equalisation and Space Time Coding for Transmission over Wireless channels. New York, USA: John Wiley-IEEE Press, 2002.

[3] C. Berrou, A. Glavieux and P. Thitimajshima, "Near Shannon Limit Error-Correcting Coding and Decoding: Turbo Codes," in Proceedings of the International Conference on Communications, (Geneva, Switzerland), pp. 1064-1070, May 1993.

[4] P. Robertson, T. Wörz, "Bandwidth-Efficient Turbo Trellis-Coded Modulation Using Punctured Component Codes," IEEE Journal on Selected Areas in Communications, vol. 16, pp. 206-218, February 1998.

[5] J. Y. Chung, S. X. Ng and L. Hanzo, "Burst-by-burst adaptive coded modulation-aided joint detection-based CDMA for wireless video telephony," in IEEE Vehicular Technology Conference, (Birmingham, USA), pp. 1317-1321, May 2002.

[6] ITU-T, "Recommendation H.263: Video Coding for Low Bitrate communication," March 1998.
[7] Special Mobile Group of ETSI, "UMTS: Selection procedures for the choice of radio transmission technologies of the UMTS," tech. rep., European Telecommunications Standard Institute (ETSI), France, 1998.

[8] L. Hanzo, P.J. Cherriman and J. Streit, Wireless Video Communications: Second to Third Generation Systems and Beyond. NJ, USA: IEEE Press., 2001.

[9] R. Gallager, "Low Density Parity Check Codes," IRE Transactions On Information Theory, pp. 21-28, January 1962.

[10] F. Guo, S. X. Ng and L. Hanzo, "LDPC assisted Block Coded Modulation for Transmission over Rayleigh Fading Channels," in IEEE Vehicular Technology Conference, (Jeju, Korea), April 2003.

[11] M. Y. Alias, F. Guo, S. X. Ng, T. H. Liew and L. Hanzo, "LDPC and Turbo Coding Assisted Space-Time Block Coded OFDM," in IEEE Vehicular Technology Conference, (Jeju, Korea), April 2003.

[12] L. Hanzo, C.H. Wong, and M.S. Yee, Adaptive Wireless Tranceivers. Chichester, UK: John Wiley-IEEE Press, 2002.

[13] L. Hanzo, L.L. Yang, E.L. Kuan and K. Yen, Single- and Multi-Carrier CDMA. Chichester, UK: John Wiley-IEEE Press, 2003.

[14] P. Jung and J. Blanz, "Joint detection with coherent receiver antenna diversity in CDMA mobile radio systems," IEEE Transactions on Vehicular Technology, vol. 44, pp. 76-88, Feb. 1995.

[15] ITU-T Rec. H.26L/ISO/IEC 11496-10, "Advanced Video Coding," September 2002.

[16] P. Robertson, E. Villebrun and P. Höher, "A Comparison of Optimal and Sub-Optimal MAP Decoding Algorithms Operating in Log Domain," in Proceedings of the International Conference on Communications, pp. 1009-1013, June 1995.

[17] M.C. Davey, "Error-correction using low density parity check codes," Ph.D thesis, University of Cambridge,UK, 1999.

[18] A. Klein, G. K. Kaleh, and P. W. Baier, "Zero forcing and minimum mean square error equalization for multiuser detection in code division multiple access channels," IEEE Transactions on Vehicular Technology, vol. 45, pp. 276-287, May 1996.

[19] G. H. Golub and C. F. van Loan, Matrix Computations. North Oxford Academic, 1983

[20] G. Bjontegaard, T.Wiegand, "H.26L Test Model Long Term Number 8 (TML-8) draft 0," June 2001. 